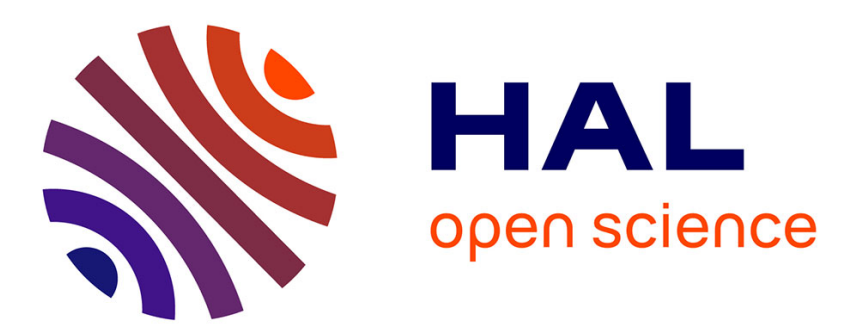

\title{
A relationship between stabilized finite element methods and the Galerkin method with bubble functions
}

\author{
F. Brezzi, Marie-Odile Bristeau, L.P. Franca, Michel Mallet, G. Roge
}

\section{To cite this version:}

F. Brezzi, Marie-Odile Bristeau, L.P. Franca, Michel Mallet, G. Roge. A relationship between stabilized finite element methods and the Galerkin method with bubble functions. [Research Report] RR-1428, INRIA. 1991. inria-00075132

\section{HAL Id: inria-00075132 \\ https://hal.inria.fr/inria-00075132}

Submitted on 24 May 2006

HAL is a multi-disciplinary open access archive for the deposit and dissemination of scientific research documents, whether they are published or not. The documents may come from teaching and research institutions in France or abroad, or from public or private research centers.
L'archive ouverte pluridisciplinaire $\mathbf{H A L}$, est destinée au dépôt et à la diffusion de documents scientifiques de niveau recherche, publiés ou non, émanant des établissements d'enseignement et de recherche français ou étrangers, des laboratoires publics ou privés. 


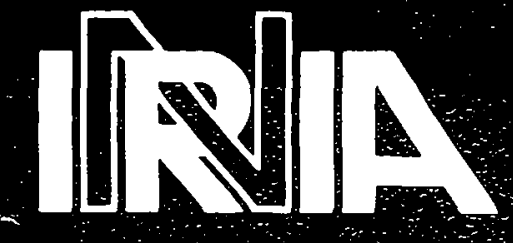

UNITÉ DE RECHERCHE INRIA-ROCOUENCOURT

Institut National de Recherche en Informatique et en Automatique

_.: Domaine de Voluceâu

Rocquéencourt B.P. 105

3.70153Le Chesnay Cedêx

\section{France}

Tél (1) 39635511

\section{Rapports de Recherche}

$$
N^{\circ} 1428
$$

\section{Programme 6}

Calcul Scientifique, Modélisation et

Logiciels numériques
A RELATIONSHIP BETWEEN STABILIZED FINITE ELEMENT METHODS AND THE GALERKIN METHOD WITH BUBBLE FUNCTIONS

Franco BREZZI Marie-Odile BRISTEAU Leopoldo P. FRANCA Michel MALLET Gilbert ROGÉ 
$\therefore$ 


\title{
A Relationship Between Stabilized Finite Element Methods and the Galerkin Method with Bubble Functions
}

\author{
Franco Brezzi \\ Instituto di Analisi Numerica del C.N.R. (IAN/CNR) \\ Corso Carlo Alberto, 5 \\ 27100 Paria - Italy
}
Marie-Odile Bristeau
Institut National de Recherche en Informatique et en Automatique (NRIA)
B.P. 105 - Rocquencourt
78153 Le Chesnay Cedex - France

\author{
Leopoldo P. Franca \\ Laboratório Nacional de Computação Cientifica ( $\mathrm{NCC} / \mathrm{CNPq}$ ) \\ Rua Láuro Müller 455 \\ 22290 Rio de Janeiro, RJ - Brazil
}
Michel Mallet and Gilbert Rogé
Dassault Ariation
7S, quai M. Dassault
92214 Saint-Cloud - France

To appear in

Computer Methods in Applied Mechanics and Engineering 


\title{
A Relationship between Stabilized Finite Element Methods and the Galerkin Method with Bubble Functions
}

\begin{abstract}
:
A relation between stabilized finite clement methods and the Galerkin method employing interpolations with bubble functions is established for the advective-diffusive model and for the lincarized compressible Navier-Stokes equations. The bubble functions are shown to help in stabilizing the advective operator without recourse to upwinding or any other numerical strategy. In particular, for the advective-diffusive model, the Galerkin method cmploying piecewise linears with bubble functions is shown to be equivilent to the Streamline-Upwind/Pctrov-Galerkin (SUPG) method in the diffusive limit.
\end{abstract}

Une Relation entre les Méthodes d'Eléments Finis

Stabilisées et la Méthode de Galerkin avec Fonctions Bulles

\section{Résumé :}

On établit, pour l'équation de diffusion-convection et pour les équations de NavicrStokes compressibles linéarisées, un lien entre les méthodes d'éléments finis stabilisées et la méthode de Galerkin associée à des approximations avec fonctions bulles. On montre que les fonctions bulles aident à stabiliser l'opérateur de convection sans utiliser ni décentrage, ni autres artifices numériques. En particulier, pour l'équation modèle de diffusion-convection, on démontre que la méthode de Galerkin utilisant des fonctions linéaires par morceaux plus des fonctions bulles est équivalente à la méthode SLPG (Streamline-Upwind/Petrov-Galerkin) quand le terme de diffusion est prédominant. 


\section{The Advective-Diffusive Model}

Let us first consibur the (homogeneous-Dirichlet) steady advective-diffusive problem which consists in finding the scalar field $u=u(\mathbf{x})$, such that

$$
\begin{gathered}
\mathbf{a} \cdot \nabla u-\nabla \cdot(\kappa \nabla u)=f \quad \text { in } \Omega \\
u=0 \quad \text { on } \Gamma
\end{gathered}
$$

where the given data are: the velocity field $\mathbf{a}(\mathbf{x})$, the diffusivity $\kappa=\kappa(\mathbf{x})>0$ and the source function $f(x)$. Herein $\Omega \subset \mathbb{R}^{2}$, is a bounded domain with a polygonal boundary $\Gamma$.

A standard variational formulation of (1) can be written as: Find $u \in H_{0}^{1}(\Omega)$ such that

$$
(\mathbf{a} \cdot \nabla u, v)+(\kappa \Gamma u, \nabla v)=(f, v) \quad v \in H_{0}^{1}(\Omega) .
$$

To introduce finite element approximations of (3), let us consider a partition $\mathcal{C}_{h}$ of $\bar{\Omega}$ into clement s consisting of triangles in the usual way (i.e., no overlapping is allowed between any two elements of the partition, the union of all clement domains $K$ reproduces $\bar{\Omega}$, etc.). Quasiuniformity is not assumed. As usual, we denote by $P_{1}\left(K^{*}\right)$ the set of all linear functions defined on $K^{\text {. }}$.

The simplest conforming Galerkin method to approximate (3) searches a solution in $l_{1} \subset H_{0}^{1}(\Omega)$ where

$$
V_{1}=\left\{v_{1} \in H_{0}^{1}(\Omega) \mid v_{1 \mid k} \in P_{1}(K), K \in \mathcal{C}_{h}\right\}
$$

More precisely; this method searches $u_{1} \in V_{1}$ such that

$$
\left(\mathbf{a} \cdot \nabla u_{1}, v_{1}\right)+\left(\kappa \nabla u_{1}, \nabla v_{1}\right)=\left(f, v_{1}\right) \quad v_{1} \in V_{1}
$$


For uniform meshes, this method can be written as a finite-difference stencil which reproduces a centered difference approximation of the advective operator. It is well-known in the finite difference litorature that for advective-dominated fows, i.e., when the mesh-Peclet number, defined by $\mathrm{Pe}=|\mathrm{a}| h /(2 \kappa)$, is large, spurious oscillations will frequently be detected in the solution of (5). A cure to this drawback widespread in the numerical literature is that some "artificial" viscosity has to be added to the formulation. In the finite element method this is achicved throngh the use of a Petror-Galerkin formulation $[6,9]$ or by a Stabilization Method such as SLPG of Hughes and Brooks $\{5,10]$ or Streamline Diffusion of Jolmson et al. [12,13]. Heretofore, there has been a consensus that the Galerkin method could not be used to stabilize the advective operator. The reasoning is simple: Take $V_{h} \subset H_{0}^{1}(\Omega)$ to be a typical finite element space consisting of piccewise continuous polynomials or other sophisticated approximation. Then, as before, the method consists of finding $u_{h} \in V_{h}$ such that

$$
B\left(u_{h}, v_{h}\right)=\left(\mathbf{a} \cdot \nabla u_{h}, v_{h}\right)+\left(\kappa \nabla u_{h}, \nabla v_{h}\right)=\left(f, v_{h}\right) \quad v_{h} \in V_{h} .
$$

The stability of this method can be roughly examined by taking $v_{h}=u_{h}$ which rields

$$
B\left(u_{h}, u_{h}\right)=\left(\mathbf{a} \cdot \nabla u_{h}, u_{h}\right)+\left\|\kappa^{1 / 2} \nabla u_{h}\right\|_{0}^{2} .
$$

For a given velocity vector field with $\nabla \cdot \mathrm{a}=0$, note that by integration by parts and using the homogeneous boundary condition (2) it follows that

$$
\left(\mathbf{a} \cdot \nabla u_{h}, u_{h}\right)=0
$$

Therefore, apparently, the stability of (6) relies, in this case, entirely on $\left\|\kappa^{1 / 2} \nabla u_{h}\right\|_{0}^{2}$ which can be "small" for very "small" $\kappa$ ! From this argument, it 
scems hopcless that the Galerkin method can produce stable solutions for small r. He will see below, that this is not entirely true. For the moment let us review some stabilized methods for this equation:

i) Classical Lipuind:

$$
B_{L}\left(u_{h}, v_{h}\right)=\left(\mathbf{a} \cdot \nabla u_{h}, v_{h}\right)+\left((\kappa+h) \nabla u_{h}, \nabla v_{h}\right)=\left(f, v_{h}\right) \quad v_{h} \in V_{h}
$$

ii) Streamline Lpwind Petror-Galerkin:

$$
\begin{aligned}
B_{S l P G}\left(u_{h}, v_{h}\right)= & \left(\mathbf{a} \cdot \Gamma u_{h}, v_{h}\right)+\left(\kappa \nabla u_{h}, \nabla v_{h}\right) \\
& +\sum_{h \in \mathcal{C}_{h}}\left(\mathbf{a} \cdot \nabla u_{h}-\nabla \cdot\left(\kappa u_{h}\right), \tau \mathbf{a} \cdot \nabla v_{h}\right)_{k} \\
= & \left(f, v_{h}\right)+\sum_{h \in \mathcal{C}_{h}}\left(f, \tau \mathbf{a} \cdot \nabla v_{h}\right)_{K},
\end{aligned}
$$

where

$$
\begin{aligned}
& \tau\left(\mathbf{x}, \operatorname{Pe}_{K^{\prime}}(\mathbf{x})\right)=\frac{h_{K^{\cdot}}}{2|\mathbf{a}(\mathbf{x})|_{p}} \xi\left(\operatorname{Pe}_{K}(\mathbf{x})\right) \\
& \operatorname{Pe}_{h} \cdot(\mathbf{x})=\frac{m_{k}:\left.\mathbf{a}(\mathbf{x})\right|_{p} h_{K}}{2 \kappa(\mathbf{x})} \\
& \xi\left(\operatorname{Pe}_{h}(\mathbf{x})\right)= \begin{cases}\operatorname{Pe}_{K}(\mathbf{x}) & , 0 \leq \operatorname{Pe}_{h}(\mathbf{x})<1 \\
1 & , \operatorname{Pe}_{h}(\mathbf{x}) \geq 1\end{cases} \\
& |\mathbf{a}(\mathbf{x})|_{p}= \begin{cases}\left(\sum_{i=1}^{\Lambda}\left|a_{i}(\mathbf{x})\right|^{p}\right)^{1 / p} & , \quad 1 \leq p<\infty \\
\max _{i=1, \Lambda}\left|a_{i}(\mathbf{x})\right| & , \quad p=\infty\end{cases} \\
& m_{k}=\min \left\{\frac{1}{3}, 2 \widetilde{C}_{k}\right\} \\
& \widetilde{C}_{k} \sum_{h \in \mathcal{C}_{h}} h_{h}^{2}\|\Delta v\|_{0, K}^{2} \leq\|\nabla v\|_{0}^{2} \quad v \in V_{h}
\end{aligned}
$$

(see Hughes and Brooks [10] for the original version of SUPG and [8] and references therein for other constructions of $\tau$ and further elaborations). 
Contrary to the Galcrkin method, these stabilized methods have improwed stahility characteristics cren for "small" $\kappa$ (or high mesh-Peclet number). Indecd, for the classical upwind method

$$
B_{U}\left(u_{h}, u_{h}\right)=\left\|(\kappa+h)^{1 / 2} \nabla u_{h}\right\|_{0}^{2}
$$

which is clearly more coercive than $B\left(u_{h}, u_{h}\right)$ for small $\kappa$. However the clascical upwind method has the following major drawbacks: i) it is too dissipative in the "crosswind direction"; ii) it is not a consistent method, i.e., the exact solution docs not satisfy ( 7 ) producing consistency errors that have to be estimated. This drawback limits the use of ( 7 ) to linear clements in the absence of sources, since a high order accurate solution is not possible, in general, by emploring high order interpolations for ( 7$)$.

For the SLPG method additional stability is obtained in the streamline direction, based on a residual formulation. This method combines improved stability with high order accuracy (i.e., high order polynomials yield better comvergence rates for sufficiently smooth solutions). Indeed, stability of SL'PG follows by:

$$
B_{S l \cdot P G}\left(u_{h}, u_{h}\right)=\left\|\kappa^{1 / 2} \nabla u_{h}\right\|_{0}^{2}+\left\|\tau^{1 / 2} \mathbf{a} \cdot \nabla u_{h}\right\|_{0}^{2}-\sum_{h \in \mathcal{C}_{h}}\left(\Gamma \cdot\left(\kappa \nabla u_{h}\right), \tau \mathbf{a} \cdot \nabla u_{h}\right)_{h}
$$

where by the design of $\tau$, for $\mathrm{Pe}_{k} \geq 1$

$$
\tau=\frac{h_{K}}{2|\mathbf{a}(\mathbf{x})|_{p}} \frac{1}{\mathrm{Pe}_{K}(\mathbf{x})} \frac{m_{k}|\mathbf{a}(\mathbf{x})|_{p} h_{K}}{2 \kappa(\mathbf{x})} \leq \frac{m_{k} h_{K}^{2}}{4 \kappa(\mathbf{x})}
$$

and therefore the third term in (15) can be bounded from below as follow's (as- 
suming constant $\kappa$ )

$$
\begin{aligned}
&-\sum_{h \in C_{h}}\left(\nabla \cdot\left(\kappa \nabla u_{h}, \tau \mathbf{a} \cdot \nabla u_{h}\right)_{h}=-\sum_{h \in \mathcal{C}_{h}}\left(\tau^{1 / 2} \nabla \cdot\left(\kappa \nabla u_{h}\right), \tau^{1 / 2} \mathbf{a} \cdot \nabla u_{h}\right)_{k}\right. \\
& \geq-\frac{1}{2}\left\|\tau^{1 / 2} \mathbf{a} \cdot \nabla u_{h}\right\|_{0}^{2}-\frac{1}{2} \sum_{h \in \mathcal{C}_{h}}\left\|\tau^{1 / 2} \kappa \Delta u_{h}\right\|_{0, K}^{2} \\
& \geq-\frac{1}{2}\left\|\tau^{1 / 2} \mathbf{a} \cdot \nabla u_{h}\right\|_{0}^{2}-\frac{1}{2} \sum_{k \in \mathcal{C}_{h}} \frac{m m_{k} h_{h}^{2}}{4} \kappa\left\|\Delta u_{h}\right\|_{0, h^{\prime}}^{2} \\
& \geq-\frac{1}{2}\left\|\tau^{1 / 2} \mathbf{a} \cdot \nabla u_{h}\right\|_{0}^{2}-\frac{1}{2} \frac{m_{k}}{4 \tilde{C}_{k}} \kappa \nabla u_{h} \|_{0}^{2} \\
& \geq-\frac{1}{2}\left\|\tau^{1 / 2} \mathbf{a} \cdot \nabla u_{h}\right\|_{0}^{2}-\frac{\kappa}{4}\left\|\nabla u_{h}\right\|_{0}^{2}
\end{aligned}
$$

and therefore combining with (15) yields

$$
B_{S L i P G}\left(u_{h}, u_{h}\right) \geq C\left(\left\|\tau^{1 / 2} \mathbf{a} \cdot \nabla u_{h}\right\|_{0}^{2}+\kappa \mid\left\|\nabla u_{h}\right\|_{0}^{2}\right)
$$

Then an error estimate may be established in the "energy" norm

$$
\left(\left\|\tau^{1 / 2} \mathbf{a} \cdot \Gamma u_{h}\right\|_{0}^{2}+\kappa\left\|\Gamma u_{h}\right\|_{0}^{2}\right)^{1 / 2}
$$

Further discussion and analysis can be found in $[8,12,13]$ and references therein.

We are now ready to come back to the central point of this work. Consider the Galerkin method given by (6) with $V_{h}=V_{1}^{b}$ where

$$
V_{1}^{b}=\left\{v \in H_{0}^{1}(\Omega) \mid v_{\mid K} \in P_{1}\left(K^{*}\right) \in B\left(K^{*}\right), K \in \mathcal{C}_{h}\right\}
$$

and $B\left(K^{*}\right)$ denotes standard bubble functions defined on $K$ with the following properties

$$
\begin{array}{lll}
\int_{K} \phi d \Omega=C_{1 K^{\prime}} h_{h}^{2}, & \phi_{\mid K} \in B\left(K^{\prime}\right), & K \in \mathcal{C}_{h} \\
\|\nabla \phi\|_{0, K}=C_{2 K^{\prime}}, & \phi_{\mid K} \in B(K), & K \in \mathcal{C}_{h}
\end{array}
$$


where $C_{1 K}$ and $C_{2 k}$ are independent of the element parameter $h_{K}$. These equations hold for the cubic bubble, for cximple.

Our result can be stated as:

THEOREXI: Assuming the given data $(a, k$ and $f)$ to be piecewise constant, then the Gaterkin method employing $V_{h}=V_{1}^{b}$ is equiwant to the SLPG method with $V_{h}=V_{i}$ for diffusive-dominated fows and appropriately defined element parameters $h_{K}$.

Proof: Let us write the solution $u_{h}$ of the Galerkin method with $V_{h}=V_{1}^{b}$ as

$$
u_{h}(x)=u_{1}(x)+\sum_{h \in \mathcal{C}_{h}} \phi(x) u_{b}
$$

where $u_{1}(x) \in V_{1}, u_{b}$ is the bubble nodal value and the bubble function $\phi_{\mid k} \in$ $B\left(K^{-}\right) \subset H_{0}^{l}\left(K^{-}\right)$satisfies

$$
\phi(x)=0 \quad \text { on } \quad \partial K, \quad K \in \mathcal{C}_{h}
$$

First consider the "bubble-cquation" obtained by taking $v=\phi_{1}$ on $K$ and $v=0$ elsewhere. Note that $v \in H_{0}^{1}\left(K^{\prime}\right) \subset H_{0}^{1}(\Omega)$. Then in $(6)$

$$
\left(\mathbf{a} \cdot \nabla u_{1}, \phi\right)_{K}+\left(\mathbf{a} \cdot \Gamma\left(\phi u_{b}\right), \phi\right)_{K}+\left(\kappa \nabla u_{1}, \nabla \phi\right)_{K^{*}}+\left(\kappa \nabla\left(\phi u_{b}\right), \Gamma \phi\right)_{K}=(f: \dot{\phi})_{K}
$$

However, under the hypothesis of piecewise constant data, by integration-by-parts the second and third terms above are zero. Indeed,

$$
\begin{gathered}
(\mathbf{a} \cdot \nabla \phi, \phi)_{K}=-(\phi, \mathbf{a} \cdot \nabla \phi)_{K}-\left(\phi^{2}, \nabla \cdot \mathbf{a}\right)_{K}+\left(\mathbf{a} \cdot \mathbf{n}, \phi^{2}\right)_{\partial K} \\
\left(\kappa \nabla u_{1}, \nabla \phi\right)_{K}=-\left(\nabla \cdot\left(\kappa \nabla u_{1}\right), \phi\right)_{K}+\left(\kappa \nabla u_{1} \cdot \mathbf{n}, \phi\right)_{\partial K}
\end{gathered}
$$


and for piccewisc-constant $\mathbf{a}$ and $\kappa, \nabla \cdot \mathbf{a}=0$ on $\kappa$, and $\nabla \cdot\left(\kappa \nabla u_{1}\right)=\kappa \Delta u_{1}=0$ on $K ;$ and since $\phi=0$ on $\partial K$ then the assertion follows. Therefore the bubble cquation (23) sinplifics to:

$$
u_{b}(\kappa \nabla \phi, \nabla \phi)_{k}=\left(f-\mathbf{a} \cdot \Gamma u_{\jmath}, \phi\right)_{k}
$$

Since $f$ and a are piccewise-constants by hypothesis and since $\nabla u_{1}$ is also a piecewise-constant function, then we may pull $f-\mathbf{a} \cdot \nabla u_{1}$ out of the right-hand-side integral and write the bubble nodal value as

$$
u_{b}=\frac{-1}{\kappa_{\mid k}\|\Gamma\|_{0, K}^{2}}\left(\mathbf{a} \cdot \Gamma u_{1}-f\right)_{\mid k} \int_{K^{\prime}} \phi d \Omega .
$$

Let us now consider $v=v^{\prime}{ }_{1} \in V_{1}$. Then in (6):

$$
\left(\mathbf{a} \cdot \Gamma u_{1}, v_{1}\right)+\sum_{k \in \mathcal{C}_{h}}\left(\mathbf{a} \cdot \nabla\left(\phi u_{b}\right), v_{1}\right)_{k}+\left(\kappa \nabla u_{1}, \nabla v_{1}\right)=\left(f, v_{1}\right)
$$

(We have used the fact that $\left(\kappa \nabla \phi, \nabla_{v_{1}}\right)_{K}=0, H \in \mathcal{C}_{h}$, as in (25)).

Note that, cxtracting the sccond term from (2S). we obtain the Galertin method employing linear elements, cf. eq. (5). Let us now examine the second term of $(2 \varepsilon)$. For each element $K$ we have:

$$
\begin{aligned}
\left(\mathbf{a} \cdot \Gamma\left(\phi u_{b}\right), v_{1}\right)_{k} & =-u_{b}\left(\phi, \mathbf{a} \cdot \nabla v_{1}\right)_{K} \\
& =+\frac{1}{\kappa_{\mid K}\|\Gamma \phi\|_{0, K}^{2}}\left(\mathbf{a} \cdot \Gamma u_{1}-f\right)_{\mid K} \cdot\left(\mathbf{a} \cdot \Gamma v_{1}\right)_{\mid K} \cdot\left(\int_{K} \phi d \Omega\right)^{2} .
\end{aligned}
$$

Using the properties of the standard bubble function (eq.(19)) we can rewrite

$$
\int_{K^{\prime}} \phi d \Omega=C_{3 K^{\prime}} \text { Measure }\left(K^{\prime}\right)
$$

and pull back the piecewise-constant values to an integral on the element interior, i.e.,

$$
\left(\mathbf{a} \cdot \nabla\left(\phi u_{b}\right), v_{1}\right)_{K}=\frac{C_{1 K} C_{3 K}}{C_{2 K^{*}}^{2}} \frac{h_{K^{*}}^{2}}{\kappa_{\mid K^{\prime}}}\left(\mathbf{a} \cdot \nabla u_{1}-f, \mathbf{a} \cdot \nabla v_{1}\right)_{K^{\prime}} .
$$


The"cfore rombining this result with $(28)$ yields the SUPG method using P1 approximation with a cocfficient $\tau$ of $O\left(h_{k}^{2} / \kappa_{\mid k}\right)$, which is the order of the paraneter in the SUPG for diffusive-dominated flows.

Consider the mumerical test discussed, e.g., in $[8,10]$ for a moderately advection-dominated flow skew to the mesh. Taking a regular mesh with $h=0.05$, and given data $\kappa=0.005$ and $|a|_{2}=1$ we have by (10) for a linear element $(k=1), \mathrm{Pe}=\frac{1}{3} \times 1 \times 0.05 /(2 \times 0.005)=5 / 3>1$. Elevation plots of $u$ are shown in Figure 1 for the Galerkin method using linear elements and linear elements with bubbles. Note that similarly to the SLPG method, the Galerkin method with bubbles does not present spurious oscillations in smooth regions. For the linear element case, oscillations propagate even in smooth regions.

\section{REMARKS:}

1. The THEOREM shows that the Galerkin method employing $V_{h}=l_{1}^{b}$ tas the improred stability characteristics of "artificial" diffusion in the streamline direction. This is a rather surprising result at first sight, since the bubble function, that induces this feature, is a perfectly symmetric test and trial function and does not have any "irick" built-in a priori. It comes out of its condensation that the linear support of the $V_{1}^{b}$-space has similar convergence properties of the SUPG method for low mesh-Peclet numbers. From uhat we know of the SUPG method, there is no reason to compute the bubble nodal value afterwards, since the vertex nodal values produce a desirable numerical solution. We should note that the relationship between bubbles and SLPG was first noted in G. Rogé's thesis [17] (see also [4]) for a one-dimensional model in absence of source. For one dimension the effect of artificial diffusion could be 
vicwed as classical upwinding (no preferential direction) or as (degencrated) streamline upwinding. By going to the multidinnensional case, it becomes clear that the bubbles do lave the stremmline diffusion properties of the SLPG nucthod.

2. Once the result is understood, the next question is what to do when $r \rightarrow 0$. In other words, when the mesh-Peclet number is large, will the Galerhin mothod with bubbles perform satisfactorily? In the proof of the THEOREM the condensation procedure is shown in (26), which indicates that there will be a division by $(\kappa \nabla \phi, \nabla \phi)_{k}$. One possibility to overcome the indesirable effect when $\kappa \rightarrow 0$ is to simply replace $(\kappa \nabla \phi, \nabla \phi)_{K}$ by $C_{4 k} \mid \mathbf{a}_{p_{p}} h_{K}$ and proceed as before. Clearly in this case consistency is not affected, since the bubble term now becomes

$$
\left(\mathbf{a} \cdot \nabla\left(\phi u_{b}\right), v_{1}\right)_{h}=\frac{C_{1 K} C_{3 K}}{C_{4 K}} \frac{h_{K}}{|\mathbf{a}|_{p}}\left(\mathbf{a} \cdot \nabla u_{1}-f, \mathbf{a} \cdot \nabla v_{1}\right)_{K^{\prime}} .
$$

In other words, SLPG can be completely recovered by this procedure with a parameter $O\left(h_{k} /|\mathbf{a}|_{p}\right)$ when $\kappa \rightarrow 0$.

3. The solution adrocated in Remark 2 can be found alternatively by redefining the bubble functions. Consider, for example, a strip crossing a given triangle in the mesh, through its barycenter. The strip is constructed to have the width of $\kappa /|\mathbf{a}|_{p}$. Its length is approximately equal to the element diameter $h_{h}$. Inside the strip we let the bubble value go linearly from its value along the border of the strip (approximately one near the barycenter) to zero in the mid-line parallel to the length of the strip. This construction implies that the area of the strip is $C h_{K} \kappa /|\mathbf{a}|_{p}$ and the Euclidean norm of the gradient of the 
bubble is approximately $|\mathbf{a}|_{p} / \mathrm{h}$. Therefore

$$
\begin{aligned}
(\kappa \Gamma \phi, \Gamma \phi)_{\kappa} & \cong C \kappa\left(\frac{|\mathbf{a}|_{p}}{\kappa}\right)^{2} \frac{h_{h \kappa}}{|\mathbf{a}|_{p}} \\
& =C|\mathbf{a}|_{p} h_{K}
\end{aligned}
$$

and we achieved the same goal as in the previous remark, since the integrals $\int_{h} \phi d \Omega, h^{-} \in \mathcal{C}_{h}$ are still $O\left(h_{h}^{2}\right)$ by this construction.

4. Let us consider in this remark a const ant diffusivity $\kappa$. By multiplying equation (28) by $\kappa$ and letting $\kappa \rightarrow 0$, we obtain the least-squares method for hyperbolic equations. If we wish to compute with this method in this limit $(\kappa \rightarrow 0)$, then in the implementation divisions by small numbers should be aroided in the condensation of the bubbles. One way to carry this out is to set $\kappa=1$ in eq. (27) and multiply the first, third and fourth terms of (2S) by $\kappa$.

5. Pierre [14.15] and Bank and Welfert [2] have established, for the Stokes problem, the re'ation between a Galerkin method with bubble furctions (the socalled Miri-element of Arnold, Brezzi and Fortin [1]) and the stabilized methods of Brezzi and Pitkäranta [3] and Hughes, Franca and Balestra [11]. The present work points out that bubbles help not only to fulfill compatibility between spaces as in Stokes, but also to stabilize the advective operator. This is more apparent in the next section.

\section{Linearized Compressible Navier-Stokes Equations}

Good numerical results were obtained at INRIA and Dassault Ariation (see $[4,17])$ for the simulation of the compressible Navier-Stokes equations at low Reynolds number, employing the Galerkin method with density and temperature approximated by a piecewise linear function and velocity with a piecewise linear 
plus a bubble function. The first attempts to explain this good behaviour were associated with the Stokes-like equations obtained in the limit as Reynolds number goes to zero [7,16]. However, we have secn in the previous section that, in fact, the bubble function also adds stability to the advective operator. For systems it is not clear, a priori, which variables to consider with bubble functions. We will restrict our attention to a sinplified set of linearized steady compressible NavierStokes equations, to shed some light on the improvement of stability obtained in the aforementioned works.

Let us then consider the problem of finding the density $\rho$, the velocity $u$ and the temperature $T$ such that

$$
\begin{gathered}
\mathbf{u}_{o} \cdot \nabla \rho+\rho_{o} \nabla \cdot \mathbf{u}=\mathbf{0} \\
(\Gamma \mathbf{u}) \mathbf{u}_{o} \div(\jmath-1)\left(\frac{T_{o}}{\rho_{o}} \nabla \rho+\nabla T\right)-\frac{1}{\operatorname{Re} \rho_{o}}\left[\Delta \mathbf{u}+\frac{1}{3} \nabla(\Gamma \cdot \mathbf{u})\right]=\mathbf{f} \\
\mathbf{u}_{o} \cdot \nabla T+(\gamma-1) T_{o} \Gamma \cdot \mathbf{u}-\frac{\gamma}{\operatorname{Re} \rho_{o} \operatorname{Pr}} \Delta T=0
\end{gathered}
$$

where $\rho_{0}, \mathbf{u}_{o}$ and $T_{0}$ are given piecewise constant fields, $\gamma$ is the ratio betwcen specific heats. Re is the Reynolds number and Pr is the Prandtl number. The dissipation due to viscous terms have been neglected in equation (34) for keeping simple the arguments that follow. For external flows we let $\Gamma_{\infty}$ be the far-field boundary of the domain and $\Gamma_{B}$ the boundary of a rigid body immersed in the flow. Denoting by

$$
\begin{aligned}
& \Gamma_{\infty}^{-}=\left\{x \in \Gamma_{\infty} \mid \mathbf{u}_{\infty} \cdot \mathbf{n}<0\right\} \\
& \Gamma_{\infty}^{+}=\Gamma /\left(\Gamma_{\infty}^{-} \cup \Gamma_{B}\right)
\end{aligned}
$$

where $\mathbf{u}_{\infty}$ is the free stream velocity and $\mathbf{n}$ is the outuard unit vector to $\Gamma$. To (32)-(34) we append the following set of boundary conditions:

$$
\rho=\rho_{p} \quad \text { on } \Gamma_{\infty}^{-}
$$




$$
\begin{array}{ll}
\mathbf{u}=\mathbf{u}_{p} & \text { on } \Gamma_{B} \cup \Gamma_{\infty}^{-} \\
T=T_{p} & \text { on } \Gamma_{B} \cup \Gamma_{\infty}^{-}
\end{array}
$$

and

$$
\begin{gathered}
\left(\Gamma \mathbf{u}+\frac{1}{3}(\nabla \cdot \mathbf{u}) \mathbf{I}\right) \mathbf{n}=\mathbf{0} \quad \text { on } \Gamma_{\infty}^{+} \\
\Gamma T \cdot \mathbf{n}=0 \quad \text { on } \Gamma_{\infty}^{+}
\end{gathered}
$$

where $\rho_{p}, \mathbf{u}_{p}$ and $T_{p}$ are prescribed functions at the boundaries and $\mathbf{I}$ is the idertity tensor.

Let us consider the following finite element spaces:

$$
\begin{aligned}
& l_{p}^{\cdot}=\left\{\dot{\varphi} \in H^{1}(\Omega)|\varphi| K^{\prime} \in P_{1}\left(K^{\circ}\right), K^{\prime} \in \mathcal{C}_{h}, \varphi=\rho_{p} \text { on } \Gamma_{\infty}^{-}\right\} \\
& \mathbf{V}_{p}^{b}=\left\{\mathbf{v} \in\left(H^{1}(\Omega)\right)^{N} \mid \mathbf{v}_{\mid K} \in\left(P_{1}\left(K^{-}\right) \in B\left(K^{\prime}\right)\right)^{N^{\prime}}, K^{\cdot} \in \mathcal{C}_{h}, \mathbf{v}=\mathbf{u}_{p} \text { on } \Gamma_{B} \cup \Gamma_{\infty}^{-}\right\} \\
& \mathbb{H}_{p}^{-}=\left\{\theta \in H^{1}(\Omega) \mid \theta_{\mid K} \in P_{1}\left(K^{*}\right), H^{*} \in \mathcal{C}_{h}, \theta=T_{p} \text { on } \Gamma_{B} \cup \Gamma_{\infty}^{-}\right\}
\end{aligned}
$$

and $L_{0}, V_{o}^{b} . H_{o}$ which are the spaces obtained by setting to zero the boundary conditions in (40)-(42). The Galerkin method we wish to study consists in searching for $\left\{\rho_{h}, \mathbf{u}_{h}, T_{h}\right\} \in U_{p} \times \mathbf{V}_{p}^{b} \times W_{p}$ such that

$$
\begin{gathered}
\left(\mathbf{u}_{o} \cdot \nabla \rho_{h}, \varphi\right)+\left(\rho_{o} \Gamma \cdot \mathbf{u}_{h}, \varphi\right)=0 \quad \xi \in U_{o} \\
\left(\left(\Gamma \mathbf{u}_{h}\right) \mathbf{u}_{o}, \mathbf{v}\right)+(\gamma-1)\left(\frac{T_{o}}{\rho_{o}} \Gamma \rho_{h}+\nabla T_{h}, \mathbf{v}\right) \\
+\frac{1}{\operatorname{Re} \rho_{o}}\left[\left(\Gamma \mathbf{u}_{h}, \nabla \mathbf{v}\right)+\frac{1}{3}\left(\Gamma \cdot \mathbf{u}_{h}, \nabla \cdot \mathbf{v}\right)\right]=(\mathbf{f}, \mathbf{v}) \quad \mathbf{v} \in \mathbf{V}_{o}^{b} \\
\left(\mathbf{u}_{o} \cdot \nabla T_{h}, \theta\right)+(\gamma-1)\left(T_{o} \nabla \cdot \mathbf{u}_{h}, \theta\right)+\frac{\gamma}{\operatorname{Re} \rho_{o} \operatorname{Pr}}\left(\nabla T_{h}, \Gamma \theta\right)=0 \quad \theta \in W_{o}
\end{gathered}
$$


Similarly to the proof of the THEOREM in the last section, we wish to show that the Galerkin method defined in exuations (43) to (45) is equivalent to a stabilization procedure for the lincarized Navier-Stokes equations. To this end the first step is to "statically condensate" the bubble from the momentum equation. Let us decompose the trial function for the velocity as

$$
\mathbf{u}_{h}(x)=\mathbf{u}_{1}(x)+\sum_{h \in \mathcal{C}_{h}} \phi(x) \mathbf{u}_{b}
$$

Next let us take $\mathbf{v}=\phi e_{i}$, on $K$, for $i=1, \ldots, N$, and zero clscwhere, where $\mathbf{e}_{i}$ is the unity cartesian vector with components $\left(\mathbf{e}_{i}\right)_{j}=\delta_{i j}$ for $j=1, \ldots, N$. For this choice of weighting functions and replacing (46) in (44) we get:

$$
\begin{aligned}
& \left(\left(\Gamma \mathbf{u}_{1}\right) \mathbf{u}_{o}, c \mathbf{e}_{i}\right)_{K}+(\jmath-1)\left(\frac{T_{o}}{\rho_{o}} \nabla \rho_{h}+\Gamma T_{h}, \phi \mathbf{e}_{i}\right)_{h}+\frac{1}{\operatorname{Re} \rho_{o}}\left[\left(\nabla\left(\phi \mathbf{u}_{b}\right), \Gamma\left(\phi \mathbf{e}_{i}\right)\right)_{K}\right. \\
& \left.+\frac{1}{3}\left(\Gamma \cdot\left(c \cdot \mathbf{u}_{b}\right), \nabla \cdot\left(\phi \mathbf{e}_{i}\right)\right)_{h}\right]=\left(\mathbf{f}, \phi \mathbf{e}_{i}\right)_{k} \quad i=1, \ldots, \lambda \text {. }
\end{aligned}
$$

In obtrining ( $4 \bar{T})$, we have used the facts that

$$
\begin{gathered}
\left(\Gamma\left(\phi \mathbf{u}_{b}\right) \mathbf{u}_{o}, \phi \mathbf{e}_{i}\right)_{h}=0 \\
\left(\Gamma \mathbf{u}_{1}, \Gamma\left(\phi \mathbf{e}_{i}\right)\right)_{k}=0 \\
\left(\Gamma \cdot \mathbf{u}_{1}, \Gamma \cdot\left(\phi \mathbf{e}_{i}\right)\right)_{k}=0
\end{gathered}
$$

by integration-by-parts. Also note that

$$
\begin{aligned}
\left(\nabla\left(\phi \mathbf{u}_{b}\right) \cdot \nabla\left(\phi \mathbf{e}_{i}\right)\right)_{h}+ & \frac{1}{3}\left(\nabla \cdot\left(\phi \mathbf{u}_{b}\right) \cdot \nabla \cdot\left(\phi \mathbf{e}_{i}\right)\right)_{h} \\
& =\mathbf{u}_{b} \cdot\left[\int_{h}\left(\Gamma \phi \cdot \nabla \phi \mathbf{e}_{i}+\frac{1}{3}(\Gamma \phi \otimes \Gamma \phi) \mathbf{e}_{i}\right) d \Omega\right] \\
& =\left[\int_{h^{*}}\left(\nabla \phi \cdot \nabla \phi \mathbf{I}+\frac{1}{3} \nabla \phi \otimes \nabla \phi\right) d \Omega\right] \mathbf{u}_{b} \cdot \mathbf{e}_{\mathbf{i}}
\end{aligned}
$$


Therefore (47) becomes

$$
\frac{1}{\operatorname{Re} \rho_{o}} \Lambda \mathbf{u}_{b} \cdot \mathbf{e}_{\mathbf{i}}=-\left(\left(\Gamma \mathbf{u}_{1}\right) \mathbf{u}_{o}+(\gamma-1)\left(\frac{T_{0}}{\rho_{o}} \Gamma \rho_{h}+\Gamma T_{h}\right)-\mathbf{f}, \zeta \mathbf{e}_{i}\right)_{K}
$$

where

$$
\mathbf{A}=\int_{h}\left(\nabla \phi \cdot \nabla \phi \mathbf{I}+\frac{1}{3} \nabla \phi \Theta \nabla \phi\right) d \Omega
$$

Or, using the piecewise constant data assumption

$$
\mathbf{u}_{b}=-\operatorname{Re} \rho_{o} \mathbf{A}^{-1}\left(\left(\Gamma \mathbf{u}_{1}\right) \mathbf{u}_{o}+(\gamma-1)\left(\frac{T_{o}}{\rho_{o}} \nabla \rho_{h}+\nabla T_{h}\right)-\mathbf{f}\right)_{\mid K} \int_{h^{-}} \phi d \Omega
$$

Once we have computed the bubble nodal values, let us examine the effect of the decomposition (46) in each equilitrium equation. Starting with (43) we obtain

$$
\left(\mathbf{u}_{o} \cdot \Gamma \rho_{h} \cdot \varphi\right)+\left(\rho_{o} \nabla \cdot \mathbf{u}_{1}, \varphi\right)+\sum_{K \in \mathcal{C}_{h}}\left(\rho_{o} \nabla \cdot\left(\dot{o} \mathbf{u}_{b}\right), \varphi\right)_{K}=0 \quad \varphi \in U_{o}
$$

The first two terms of equation (55) are just the ones obtained by employing the Galerkin method with cqual-order linear elements. The third term at each clement can be written as:

$$
\begin{aligned}
\left(\rho_{o} \nabla \cdot\left(\phi \mathbf{u}_{b}\right), \varphi\right)_{K} & =-\mathbf{u}_{b} \cdot\left(\rho_{o} \phi \cdot \nabla \varphi\right)_{K} \\
& =-\mathbf{u}_{b} \cdot\left(\rho_{o} \Gamma_{\vartheta}\right)_{\mid K} \int_{K} \phi d \Omega .
\end{aligned}
$$

Substituting (46) into (44) with $v=v_{1} \in V_{o}^{1}$ leads us to:

$$
\begin{gathered}
\left(\left(\nabla \mathbf{u}_{1}\right) \mathbf{u}_{o}, \mathbf{v}_{1}\right)+\sum_{h \in \mathcal{C}_{h}}\left(\nabla\left(\phi \mathbf{u}_{b}\right) \mathbf{u}_{o}, \mathbf{v}_{1}\right)_{k}+(\gamma-1)\left(\frac{T_{0}}{\rho_{o}} \nabla \rho_{h}+\nabla T_{h}, \mathbf{v}_{1}\right) \\
+\frac{1}{\operatorname{Re} \rho_{o}}\left[\left(\nabla \mathbf{u}_{1}, \nabla \mathbf{v}_{1}\right)+\frac{1}{3}\left(\nabla \cdot \mathbf{u}_{1}, \nabla \cdot \mathbf{v}_{1}\right)\right]=\left(\mathbf{f}, \mathbf{v}_{1}\right) \quad \mathbf{v}_{1} \in \mathbf{V}_{o}^{1}
\end{gathered}
$$


(We have used $\left(\Gamma\left(\phi \mathbf{u}_{b}\right), \Gamma \mathbf{v}_{1}\right)_{K}=0$ and $\left(\Gamma \cdot\left(\phi \mathbf{u}_{b}\right), \nabla \cdot \mathbf{v}_{1}\right)_{K}=0$.) Except for the sccond term, all terms are present in the equal-order linear Galcrkin method. The seond term in (57) can be written at each element as:

$$
\begin{aligned}
\left(\Gamma\left(\phi \mathbf{u}_{b}\right) \mathbf{u}_{o}, \mathbf{v}_{1}\right)_{k} & =-\mathbf{u}_{b} \cdot\left(\phi,\left(\Gamma \mathbf{v}_{1}\right) \mathbf{u}_{o}\right)_{K} \\
& =-\mathbf{u}_{b} \cdot\left(\left(\Gamma \mathbf{v}_{1}\right) \mathbf{u}_{o}\right)_{\mid K} \int_{K^{*}} \phi d \Omega .
\end{aligned}
$$

Next. by substituting (46) into (45) yiclds to:

$$
\begin{gathered}
\left(\mathbf{u}_{o} \cdot \Gamma T_{h}, \theta\right)+(\gamma-1)\left(T_{o} \nabla \cdot \mathbf{u}_{1}, \theta\right)+(\gamma-1) \sum_{h \in \mathcal{C}_{h}}\left(T_{o} \nabla \cdot\left(\phi \mathbf{u}_{b}\right), \theta\right)_{h} \\
+\frac{\gamma}{\operatorname{Re} \rho_{o} \operatorname{Pr}}\left(\nabla T_{h}, \Gamma \theta\right)=0 \quad \theta \in W_{o} .
\end{gathered}
$$

Again, all terms are in the Galerkin method with equal-order linear elements, except for the third term, that can be written at each element level as:

$$
\begin{aligned}
(?-1)\left(T_{o} \Gamma \cdot\left(\phi \mathbf{u}_{b}\right), \theta\right)_{K^{\prime}} & =-(\gamma-1) \mathbf{u}_{b} \cdot\left(T_{o} \phi, \Gamma \theta\right)_{K} \\
& =-(\gamma-1) \mathbf{u}_{b} \cdot\left(T_{o} \Gamma \theta\right)_{\mid K} \int_{K} \phi d \Omega
\end{aligned}
$$

Therefore examining (55) to (60) we have shown that the Galerkin method given by equations (43) to (45) is equivalent to the equal-order linear Galerkin method plus the following perturbation terms $(P T)$, rewritten from (56). (5S) and (60) as:

$$
P T=-\sum_{k \in \mathcal{C}_{h}} \int_{k} \phi d \Omega \mathbf{u}_{b} \cdot\left(\rho_{o} \Gamma \varphi+\left(\Gamma \mathbf{v}_{1}\right) \mathbf{u}_{o}+(\gamma-1) T_{o} \Gamma \theta\right)_{\mid K^{*}}
$$

which combined with (54) gives us

$$
\begin{aligned}
P T & =\sum_{K \in \mathcal{C}_{h}}\left(\int_{K} \phi d \Omega\right)^{2} \operatorname{Re} \rho_{o} \mathbf{A}^{-1}\left(\left(\nabla \mathbf{u}_{1}\right) \mathbf{u}_{o}+(\gamma-1)\left(\frac{T_{o}}{\rho_{o}} \nabla \rho_{h}+\nabla T_{h}\right)-\mathbf{f}\right)_{\mid K^{\prime}} \\
& \cdot\left(\rho_{o} \nabla \varphi+\left(\nabla \mathbf{v}_{1}\right) \mathbf{u}_{o}+(\gamma-1) T_{o} \nabla \theta\right)_{\mid K},
\end{aligned}
$$


and by (19)

$$
\begin{aligned}
& P T=\sum_{h \in C_{h}} C_{1 k} C_{3 k} \cdot h_{h}^{2} \cdot \operatorname{Re} \rho_{o}\left(\mathbf { A } ^ { - 1 } \left(\left(\Gamma \mathbf{u}_{1}\right) \mathbf{u}_{o}+(\gamma-1)\left(\frac{T_{o}}{\rho_{o}} \Gamma \rho_{h}+\nabla T_{h}\right)\right.\right. \\
& \left.-\mathbf{f}), \rho_{o} \nabla \varphi+\left(\Gamma \mathbf{v}_{1}\right) \mathbf{u}_{o}+(\gamma-1) T_{o} \Gamma \theta\right)_{k} \\
& =\sum_{h \in C_{A}} C_{1 k} C_{3 h} \cdot h_{h}^{2} \cdot \operatorname{Re} \rho_{0}\left(\begin{array}{c}
\Gamma \rho_{h} \\
\left(\Gamma \mathbf{u}_{1}\right) \mathbf{u}_{o} \\
\nabla T_{h}
\end{array}\right)^{T} \\
& \left(\begin{array}{ccc}
(\gamma-1) T_{o} \mathbf{A}^{-1} & (\gamma-1) \frac{T_{0}}{\rho_{o}} \mathbf{A}^{-1} & (\gamma-1)^{2} \frac{T_{0}^{2}}{\rho_{o}} \mathbf{A}^{-1} \\
\rho_{0} \mathbf{A}^{-1} & \mathbf{A}^{-1} & (\gamma-1) T_{o} \mathbf{A}^{-1} \\
(\gamma-1) \rho_{o} \mathbf{A}^{-1} & (\gamma-1) \mathbf{A}^{-1} & (\gamma-1)^{2} T_{o} \mathbf{A}^{-1}
\end{array}\right)\left(\begin{array}{c}
\Gamma \varphi \\
\left(\Gamma \mathbf{v}_{1}\right) \mathbf{u}_{o} \\
\Gamma \theta
\end{array}\right) \\
& -\sum_{h \in \mathcal{C}_{h}} C_{1 h} C_{3 K^{\prime}} h_{h}^{2} \cdot \operatorname{Re} \rho_{o}\left(\mathbf{A}^{-1} \mathbf{f}, \rho_{o} \nabla \varphi+\left(\nabla \mathbf{v}_{1}\right) \mathbf{u}_{o}+(\gamma-1) T_{o} \Gamma \theta\right)_{K^{\prime}} .
\end{aligned}
$$

Therefore the perturbation terms that emanate by condensation are in fact adding a leasi-squares type form of the momentum equation, with a cocfficint $O\left(h_{h}^{2} \cdot \operatorname{Re} \rho_{0}\right)$ and the source is being corrected by the same operator.

Further examining (63) we note that the matrix

$$
\mathbf{C}=\left(\begin{array}{ccc}
(\gamma-1) T_{0} \mathbf{A}^{-1} & (\gamma-1) \frac{T_{0}}{\rho_{o}} \mathbf{A}^{-1} & (\gamma-1)^{2} \frac{T_{0}^{2}}{\rho_{o}} \mathbf{A}^{-1} \\
\rho_{0} \mathbf{A}^{-1} & \mathbf{A}^{-1} & (\gamma-1) T_{0} \mathbf{A}^{-1} \\
(\gamma-1) \rho_{0} \mathbf{A}^{-1} & (\gamma-1) \mathbf{A}^{-1} & (\gamma-1)^{2} T_{o} \mathbf{A}^{-1}
\end{array}\right)
$$

is positive-semidefinite, and therefore the perturbation terms are indeed adding a numerical dissipation. Note also that adding the viscous and thermal dissipation due to the Galerkin terms with linear elements, gives us positive contributions to the block matrices $\mathbf{C}_{22}$ and $\mathbf{C}_{33}$ rendering $\mathbf{C}$ positive definite.

Summing up, we have shown that the method given by eqs. (43)-(45) is 
equivalent to the equal-order linear Galerkin method plus a least-squares form of the momentum equation.

\section{Acknowledgement}

During part of this work Leopoldo P. Franca was partially supported by Ministere Franģais des Affaires Etraugères.

\section{References}

[1] D.N. Arnold, F. Brezzi and M. Fortin, A stable finite element for the Stokes equations. Calcolo 23 (4) (1984) 337-344.

[2] R.E. Bank and B.D. Welfert, A comparison between the mini-element and the Petrov-Galerkin formulations for the generalized Stokes problem, Comput. Methods Appl. Mech. Engrg. 83 (1990) 61-68.

[3] F. Brezzi and J. Pitkäranta, On the stabilization of finite element approximations of the Stokes problem, in: W. Hackbusch, ed., Efficient Solutions of Elliptic Systems, Notes on Numerical Fluid Mechanics, Vol. 10, pp. 11-19 (Viewig. Miesbaden, 1984).

[4] M.O. Bristeau, M. Mallet, J. Périaux and G. Rogé, Development of finite clement methods for compressible Navier-Stokes flow simulations in aerospace design, Paper AIAA-90-0403, 28th Aerospace Mceting, Jan. 90, Reno, Nerada.

[5] A.N. Brooks and T.J.R. Hughes, Streamline upwind/Petrov-Galerkin formulations for convective dominated flow's with particular emphasis on the incompressible Navier-Stokes equations, Comput. Methods Appl. Mech. Engrg. 32 (1982) 199-259. 
[6] I. Christie, D.F. Griffiths, A.R. Mitchell and O.C. Zienkicuicz, Finite element methods for sccond order differential equations with significant first derivatives, Intern. J. Numer. Methods Engrg. 10 (1976) 1359-1396.

[7] M. Fortin and A. Sonlainani, Finite clement approximation of compressible viscous flows, in H. Niki and M. Kawahara eds., Computational Methods in Flow Analysis, Vol. 2 (Ohayama University of Sciences Press, 19S8) 951-956.

[S] L.P. Franca, S.L. Frey and T.J.R. Hughes, Stabilized finite element methods: 1. Application to the advective-diffusive model, LNCC. Report \#032/90. To appear in Comput. Methods Appl. Mech. Engrg.

[9] J.C. Heinrich, P.S. Huyakorn, O.C. Zienkiewicz and A.R. Mitchell, An 'upwind' finite element scheme for two-dimensional convective transport ecuation, Intern. J. Numer. Methods Engrg. 11 (1977) 134-143.

[10] T.J.R. Hughes and A.N. Brooks, A multidimensional upwind scheme with no crosswind diffusion, in: T.J.R.Hughes, ed., Finite Elcment Methods for Convection Dominated Flows (ASME, New York, 1979) 19-35.

[11] T.J.R. Hughes, L.P. Franca and M. Balestra, A new finite element formulation for computational fuid dynamics: V. Circumventing the Babuška-Brezzi condition: A stable Petror-Galerkin formulation of the Stokes problem accomodating equal-order interpolations, Comput. Methods Appl. Mech. Engrg. $59(1956)$ \&5. 99.

[12] C. Johnson, Numerical solution of partial differential equations by the finite element method (Studentlitteratur, Sweden, 1987).

[13] C. Johnson, U. Nävert and J. Pitkäranta, Finite element methods for linear 
hyperbolic problem, Comput. Mothods Appl. Moch. Engrg. 45 (1984) 285312.

[14] R. Picre: Simple $C^{0}$ approxinations for the computation of incompressible flows, Comput. Methods Appl. Mech. Engrg. GS (19SS) 20j-227.

[15] R. Pierre, Regularization procedures of mixed finte element apuroximations of the Stckes problem, Num. Methods Partial Diff. Equations 5 (19S3) 241-258.

[16] O. Pironneau and J. Rappaz, Numerical analysis for compressible viscoms isentropic stationary flows, Publication du Laboratoire d Analyse Numerienuc de Paris 6, 1988 .

[1i] G. Rogé. On the approximation and the convergence acceleration in the finte clement numerical simulation of compressible viscous flows, Thèse de Doctorat. Université Paris 6, 1990. 


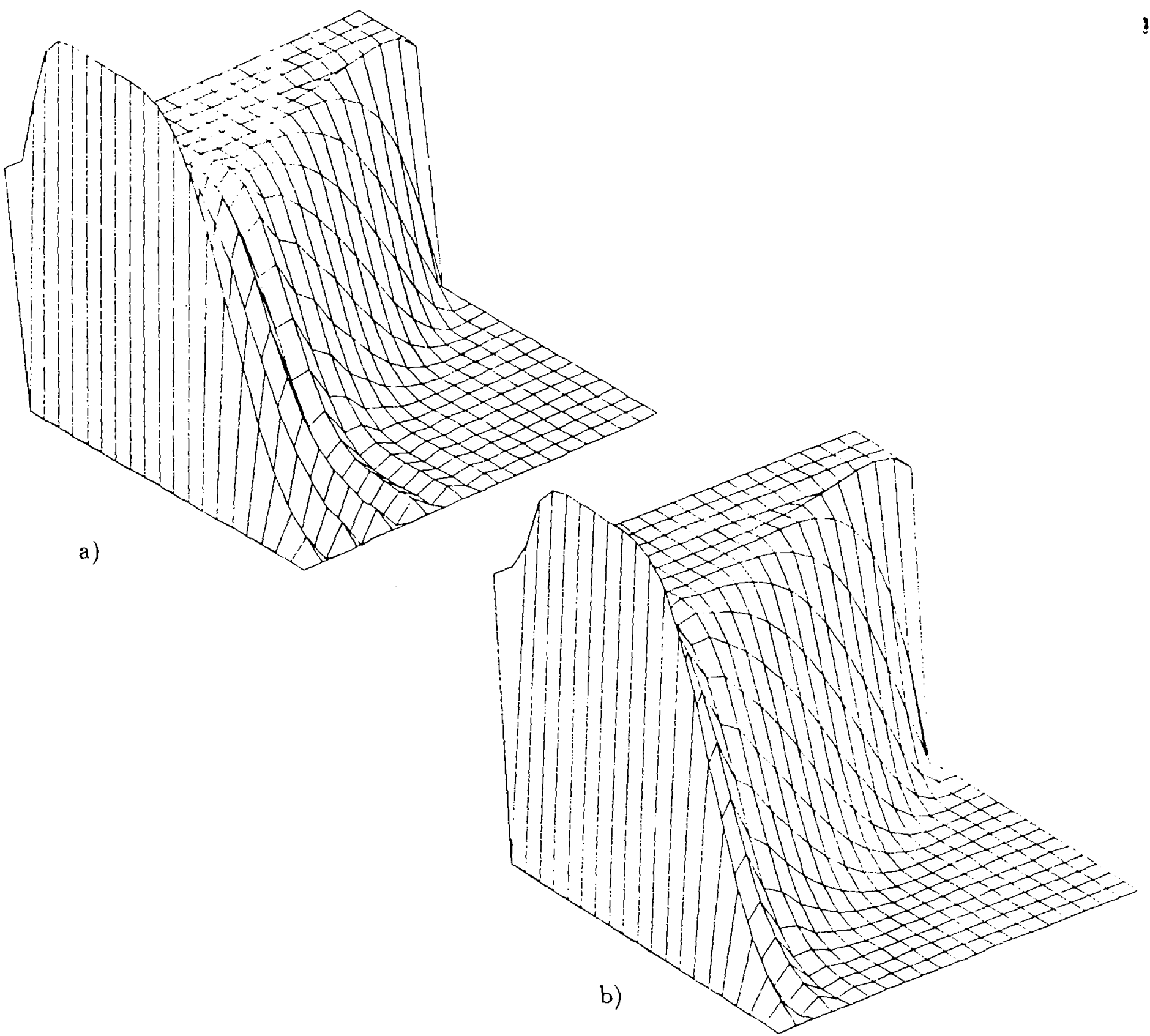

Figure 1: Advection skew to the mesh: The Galerkin method employing: a) Linear clements; b) Lincar elements with bubble functions. 
ISSN 0249 - 6399 